\title{
Analysis of Transformations Nucleated on Non-random Sites Simulated by Cellular Automata in Three Dimensions
}

\author{
Paulo Rangel Rios*, Luciana de Oliveira Pereira, Flávio Faria de Oliveira, \\ Weslley Luiz da Silva Assis, Valmir Torres de Oliveira \\ Universidade Federal Fluminense - UFF, Av. dos Trabalhadores, 420, \\ 27255-125, Volta Redonda - RJ, Brazil
}

Received October 6, 2006; Revised: April 11, 2007

\begin{abstract}
Cellular automata simulation in three dimensions is carried out to simulate microstrutural evolution for nuclei distribution ranging from a periodic arrangement to clusters of nuclei. The effect of clustering in three dimensions is found to be much more difficult to detect using conventional microstructural path analysis than in two dimensions. Microstructural path equations fit simulated data well, even when the nuclei are non-randomly located. However, the parameters obtained by means of this fitting lead to erroneous time dependent velocities. Therefore, measuring a descriptor that is sensitive to non-randomness such as the contiguity is even more important in three than in two dimensions.
\end{abstract}

Keywords: microstructure, kinetics, recrystallization, phase transformations, computer simulation, cellular automata

\section{Introduction}

Formal kinetics is often used to analyze recrystallization as well as other nucleation and growth reactions. Early formal theories of Jonhson-Mehl ${ }^{1}$, Avrami ${ }^{2-4}$ and Kolmogorov ${ }^{5}$, the JMAK theory, were subsequently expanded by DeHoff ${ }^{6}$ and further developed by Vandermeer ${ }^{7}$, resulting in the so-called microstrucural path method, MPM. In particular, this approach has been used on a routine basis to interpret results obtained for recrystallization kinetics. The usual methodology consists in measuring quantities on a planar section: the volume fraction transformed, $\mathrm{V}_{\mathrm{v}}$, and the interfacial area between transformed and untransformed regions per unit of volume, $S_{v}$, as a function of time. From these data, it is possible, by means of equations from formal kinetics, to extract information concerning the average nucleation and growth behavior of individual regions. Such analysis is usually carried out assuming that nuclei are randomly located in space, a fundamental assumption of JMAK's.

In practical situations nucleation often takes place non-random$1 y^{8,9}$. In recrystallization, for instance, the deformed state is invariably heterogeneous and preferential nucleation may take place within the regions with higher stored energy ${ }^{10}$. Non-randomness is very difficult to model by analytical methods because it is not easy to specify nuclei location if nuclei are not located in space either randomly or periodically ${ }^{11}$. Experimentally, Vandermeer ${ }^{12}$ has been advocating the use of the contiguity parameter as a useful descriptor to detect non-randomness. The contiguity, $\mathrm{C}_{\beta}$, needs measuring the interface area per unit of volume between transformed, $\beta$, and untransformed regions, $\alpha$, here designated by the symbol $\mathrm{S}_{\mathrm{v} \beta}$

$$
\mathrm{C}_{\beta}=\frac{2 \mathrm{~S}_{\mathrm{v} \beta}}{\mathrm{S}_{\mathrm{v}}+2 \mathrm{~S}_{\mathrm{v} \beta}}
$$

In recent papers, computer simulation by cellular automata ${ }^{13,14}$ has been used to investigated the kinetics in conditions that depart from randomness ${ }^{15-23}$. The simulation allows precise data to be generated, without the experimental errors. This allows to focus on the geometrical issues of the transformation and apply the theoretical results with confidence in real materials.

In a previous work, detailed cellular automata ${ }^{23}$ simulation in two-dimensions was carried out to investigate the efficiency of microstructural descriptors in assessing departure from randomness. In that work it became clear that the contiguity is very sensitive to departures from randomness. Moreover, it was found that the formal theory was able to fit the simulated results fairly well, even if the nuclei were not randomly located in space. This fitting could potentially induce serious misinterpretation of the data. This is so because the fitted parameters, obtained from non-random nuclei, could not be interpreted in their usual way.

Although the 2D simulation was interesting 3D simulations are much more relevant in practical applications. In this work, site-saturated recrystallization is simulated by using cellular automata in three dimensions in order to investigate the effect of nuclei distribution on the kinetics. In order to simplify the problem only site-saturated nucleation is considered. Formal kinetics is applied to the non-random distribution of nuclei and its validity under these circumstances is investigated. The results are compared with $2 \mathrm{D}$ simulations of previous work ${ }^{23}$. Although this work has recrystallization as main focus its results are general, valid for any nucleation and growth transformation complying with the assumptions of the simulation.

\section{Description of the Simulation}

Cellular automata methodology was used to simulate recrystallization. The implementation followed that of Hesselbarth and Göbel ${ }^{15}$ using the von Neumann neighborhood criterion. The matrix consisted of a cubic lattice with $304 \times 304 \times 304$ cells and 4096 nuclei. One cell edge was considered to have a unit length and consequently of a single cell had unit volume. The units of all quantities reported here follow from this. The number of nuclei per unit of volume, $\mathrm{N}_{\mathrm{v}}$, was kept constant and equal to $1 / 6859$. The matrix size and number of 
nuclei were chosen for reasons described elsewhere ${ }^{22}$. The nucleation was site-saturated: all nuclei appeared at $t=0$. In this work, in addition to the random nuclei distribution, the nuclei were arranged periodically and in clusters. For the periodic arrangement they were located in the center of "boxes" of $19 \times 19 \times 19$ cells. There were in total 4096 "boxes". For clustering the simulation procedure was as follows. Nucleation was allowed to occur randomly within a number of randomly selected boxes from the set of 4096 boxes mentioned above. First, 1024 boxes were randomly selected from the set of 4096 boxes, then four nuclei were randomly placed in 1024 boxes; then 256 boxes were randomly chosen and sixteen nuclei were randomly located in each box; finally, 64 boxes were randomly chosen with 64 nuclei per box. The simulation produced a sequence of matrices as a function of time. Time is discrete in CA, it takes integer values starting from $t=0$. One time unit corresponds to the interval between two consecutive matrix updates ${ }^{21,22}$. From the simulated matrices, all the desired quantities could be extracted. Hesselbarth and Göbel ${ }^{15}$ give a more detailed account of two-dimensional cellular automata in general. Oliveira ${ }^{22}$ gives further details of the three-dimensional CA simulation.

\section{Derivation of Analytical Formalism to Describe 3D Simulation}

In this section the microstructural path analysis formalism is derived for cellular automata simulations with a von Neumann neighborhood.

The basis of formal kinetics analysis is Equation 2

$$
\mathrm{V}_{\mathrm{V}}=1-\exp \left(-\mathrm{Kt}^{\mathrm{n}}\right)
$$

This equation is very "flexible" and is able to fit even complex heterogeneous transformation reasonably well. In some cases, a clear interpretation can be given to the values of $\mathrm{K}$ and $\mathrm{n}$, found by fitting the experimental data. Below we derive $\mathrm{K}$ and $\mathrm{n}$ for the specific case of cellular automata simulation of site-saturated reactions. A very general derivation of these values for spheroidal particles is given by Vandermeer et al. ${ }^{7}$.

The first point is to derive the extended volume fraction, $\mathrm{V}_{\mathrm{E}}$, that is the sum of the volumes of all growing regions supposing that they grow freely. In other words supposing that they grow as each were the only growing regions, increasing its size indefinitely without impinging on another region. First we derive the volume of one growing grain, $\mathrm{v}(\mathrm{t})$

$$
v(t)=\eta_{3}\left(\int_{0}^{t} G d t\right)^{3}
$$

where $\eta_{3}$ is a shape factor, $G$ is the interfacial velocity in extended space and $t$ is time. In a previous work ${ }^{14}$ we found that for cellular automata with $\mathrm{G}=$ constant $=1 / 3$

$$
v(t)=\frac{4}{3} t^{3}
$$

Comparing the equations gives $\eta_{3}=36$, so that the extended volume, $\mathrm{V}_{\mathrm{E}}$ is

$$
\mathrm{V}_{\mathrm{E}}(\mathrm{t})=36 \mathrm{~N}_{\mathrm{v}}\left(\int_{0}^{\mathrm{t}} \mathrm{Gdt}\right)^{3}
$$

where $\mathrm{N}_{\mathrm{v}}$ is the number of nuclei per unit of volume, $\mathrm{G}$ is the interfacial velocity in extended space and $t$ is time.

$\mathrm{G}$ can be formally described by

$$
\mathrm{G}=\frac{\mathrm{G}_{0}}{\mathrm{t}^{\mathrm{k}}}
$$

where $\mathrm{G}_{0}$ and $\mathrm{k}$ are constants. The extended volume becomes.

$$
\mathrm{V}_{\mathrm{E}}=36 \mathrm{~N}_{\mathrm{V}}\left(\frac{\mathrm{G}_{0}}{1-\mathrm{k}}\right)^{3} \mathrm{t}^{3(1-\mathrm{k})}
$$

Notice that the extended volume does not depend on nuclei location. It is the same for randomly or non-randomly located nuclei. However, the JMAK relationship between extended and real volume fraction does depend on the assumption that nuclei are randomly located in space

$$
\mathrm{V}_{\mathrm{V}}=1-\exp \left(-\mathrm{V}_{\mathrm{E}}\right)
$$

Combining this with the calculated extended volume results in:

$$
\mathrm{V}_{\mathrm{v}}=1-\exp \left(-36 \mathrm{~N}_{\mathrm{v}}\left(\frac{\mathrm{G}_{0}}{1-\mathrm{k}}\right)^{3} \mathrm{t}^{3(1-\mathrm{k})}\right)
$$

The extended area per unit of volume is also necessary for the present analysis. For a single grain, $\mathrm{s}$

$$
s(t)=\eta_{2}\left(\int_{0}^{t} G d t\right)^{2}
$$

where $\eta_{2}$ is a shape factor, In a previous work ${ }^{14}$ it was found that for cellular automata with $\mathrm{G}=$ constant $=1 / 3$

$$
\mathrm{s}(\mathrm{t})=12 \mathrm{t}^{2}
$$

Comparing the equations gives $\eta_{2}=108$, so that the extended interface area, $\mathrm{S}_{\mathrm{E}}$ is

$$
S_{E}(t)=108 N_{v}\left(\int_{0}^{t} G d t\right)^{2}
$$

Using Equation 6 for the velocity results in

$$
\mathrm{S}_{\mathrm{E}}(\mathrm{t})=108 \mathrm{~N}_{\mathrm{v}}\left(\frac{\mathrm{G}_{0}}{1-\mathrm{k}}\right)^{2} \mathrm{t}^{2(1-\mathrm{k})}
$$

Notice that, likewise the extended volume, the extended interface area does not depend on nuclei location. When nuclei are randomly located in space

$$
\mathrm{S}_{\mathrm{V}}=\mathrm{S}_{\mathrm{E}}\left(1-\mathrm{V}_{\mathrm{V}}\right)
$$

and

$$
\mathrm{S}_{\mathrm{V}}(\mathrm{t})=108 \mathrm{~N}_{\mathrm{v}}\left(\frac{\mathrm{G}_{0}}{1-\mathrm{k}}\right)^{2} \mathrm{t}^{2(1-\mathrm{k})} \exp \left(-36 \mathrm{~N}_{\mathrm{v}}\left(\frac{\mathrm{G}_{0}}{1-\mathrm{k}}\right)^{3} \mathrm{t}^{3(1-\mathrm{k})}\right)
$$

For constant velocity $\mathrm{k}=0, \mathrm{G}=\mathrm{G}_{0}$, and the expressions given in a previous work ${ }^{14}$ are recovered

$$
\begin{aligned}
& \mathrm{V}_{\mathrm{v}}=1-\exp \left(-\frac{4}{3} \mathrm{~N}_{\mathrm{v}} \mathrm{t}^{3}\right) \\
& \mathrm{S}_{\mathrm{v}}=12 \mathrm{~N}_{\mathrm{v}} \mathrm{t}^{2} \exp \left(-\frac{4}{3} \mathrm{~N}_{\mathrm{V}} \mathrm{t}^{3}\right)
\end{aligned}
$$

$\mathrm{S}_{\mathrm{V}}$ can be written as a function of $\mathrm{V}_{\mathrm{V}}$ and the "microstructural path" is obtained

$$
\mathrm{S}_{\mathrm{V}}=3\left(36 \mathrm{~N}_{\mathrm{V}}\right)^{\frac{1}{3}}\left(1-\mathrm{V}_{\mathrm{v}}\right)\left[\ln \left(\frac{1}{1-\mathrm{V}_{\mathrm{v}}}\right)\right]^{\frac{2}{3}}
$$

For site-saturated reactions, the microstructural path is independent of the interface velocity.

One way of obtaining the interface velocity is by fitting Equation 2 or 9 and 18 to the data, as will be done below. Another possibility is to obtain it directly by means of the Cahn-Hagel equation ${ }^{24}$ 


$$
\mathrm{G}_{\mathrm{CH}}=\frac{1}{\mathrm{~S}_{\mathrm{V}}} \frac{\mathrm{dV}_{\mathrm{v}}}{\mathrm{dt}}
$$

Equation 19 is a mathematically exact expression for the velocity averaged over all interface area. The interface velocity for the growth of a single grain was obtained in a previous work with the help of Equation 19

$$
\begin{aligned}
& \left.\mathrm{G}_{\mathrm{CH}} \text { (single grain }\right)=\frac{1}{\mathrm{~s}} \frac{\mathrm{dv}}{\mathrm{dt}} \cong \frac{1}{3} \\
& \mathrm{G}_{\mathrm{CH}} \text { for the simulation is reported in the next section. }
\end{aligned}
$$

\section{Results}

Figure 1 shows the simulated microstructure for randomly located nuclei: a) volume fraction equal to $0.01 \mathrm{and} b$ ) volume fraction equal to 0.1 . Figure 2 shows the simulated microstructure for clustered nuclei, 64 boxes were randomly chosen from 4096 boxes and 64 nuclei

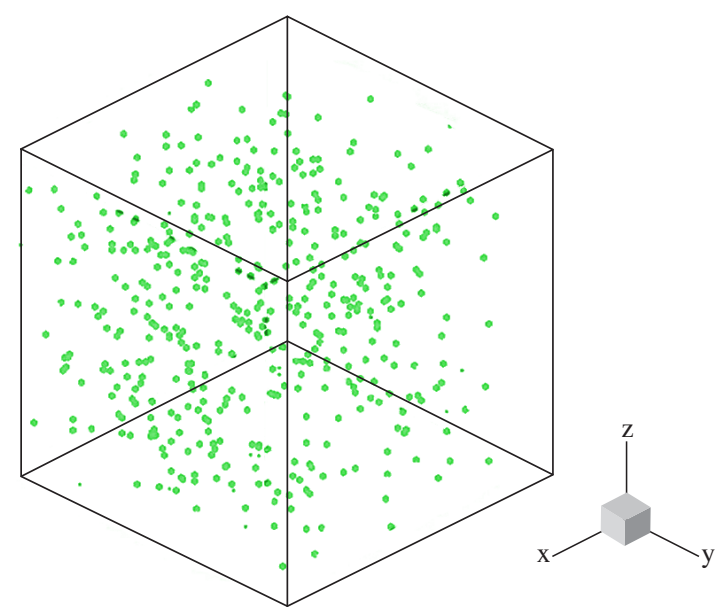

(a)

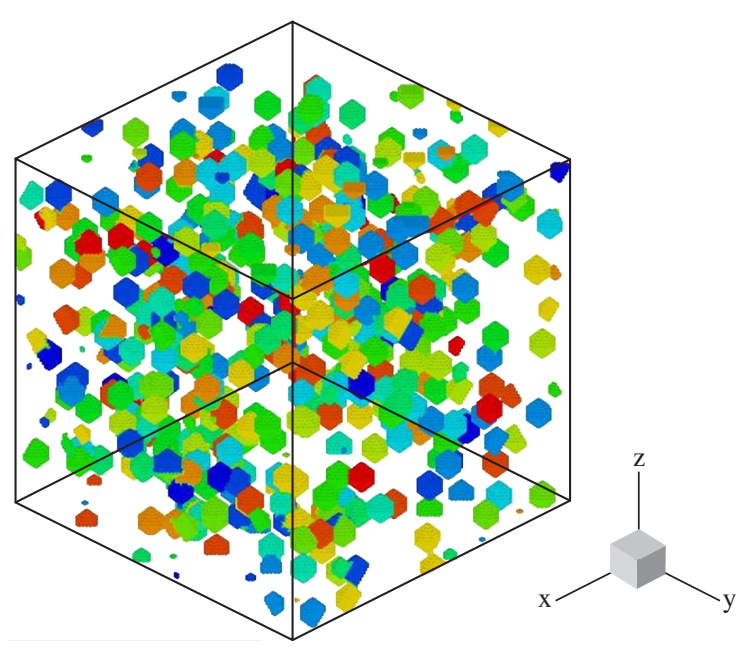

(b)

Figure 1. Simulated microstructure for randomly located nuclei: a) $t=0$ and b) volume fraction equal to 0.1 . Only part of the $304 \times 304 \times 304$ matrix is shown to avoid overloading the figure. were randomly located within each box: a) volume fraction equal to 0.01 and $b$ ) volume fraction equal to 0.1 . Figure 2 clearly shows that for clustered nuclei, impingement within the cluster takes place very rapidly and for the remaining of the growth the clustered region behaves as a single grain.

Figures 3-5 show the plots of volume fraction as a function of time; interface area per unit of volume against volume fraction, the microstructural path, and the contiguity as a function of volume fraction. In Figures 3-5 the nuclei arrangement varies from periodic to severely clustered. Three cases were chosen from Figures 3 and 4 for a more detailed microstructural path analysis to be carried out.

The general trends observed in the simulation were:

1. The more severe is the clustering the slower is the reaction kinetics relative to the kinetics of the randomly located nuclei, see Figure 3. The reaction is fastest for the periodic arrangement but, compared with 2D simulations, 3D simulations show a smaller difference between the kinetics of periodically and randomly located nuclei;

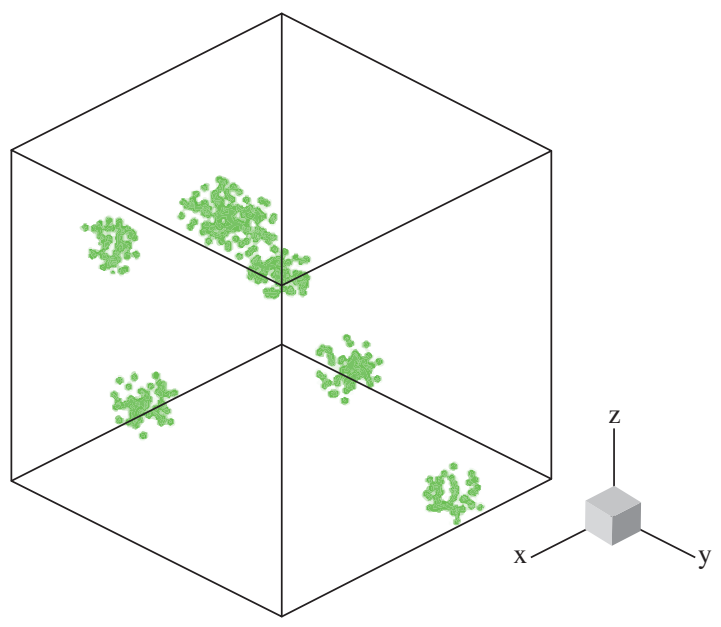

(a)

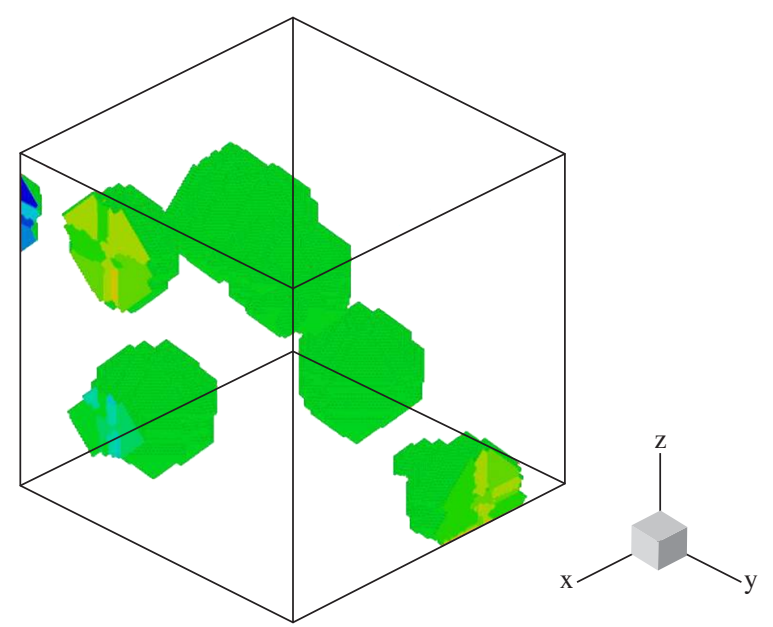

(b)

Figure 2. Simulated microstructure for clustered nuclei, 64 boxes were randomly chosen from 4096 boxes and 64 nuclei were randomly located within each box: a) $t=0$; and b) at volume fraction equal to 0.1 nuclei within clusters have already impinged. Only part of the $304 \times 304 \times 304$ matrix is shown to avoid overloading the figure. 


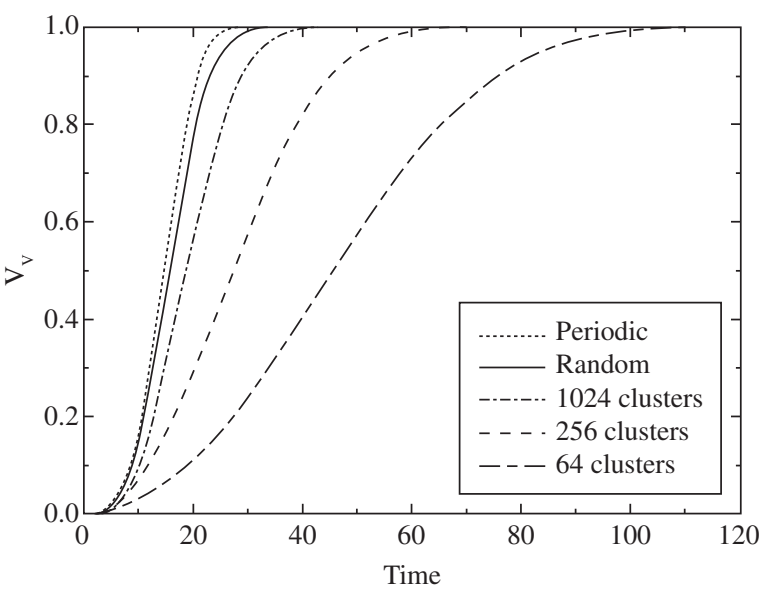

Figure 3. Volume fraction against time, simulated by CA. The reaction is faster for a periodic nuclei arrangement and slower for clusters. The number of nuclei per unit of volume is the same in all simulations.

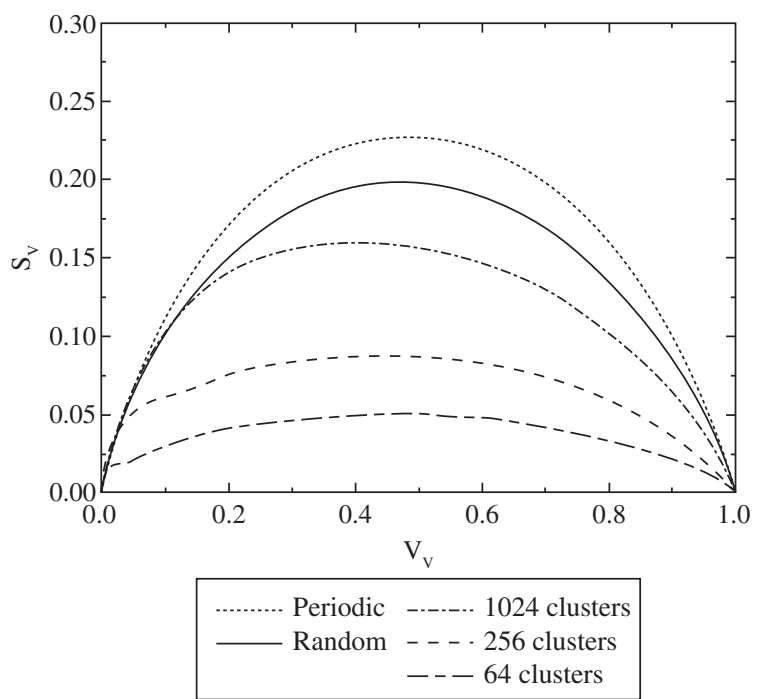

Figure 4. Interfacial area between recrystallized grains and matrix, $S_{v}$, against volume fraction, simulated by $C A$. The maximum in $S_{v}$ is more pronounced for a periodic nuclei arrangement and less pronounced for clusters.

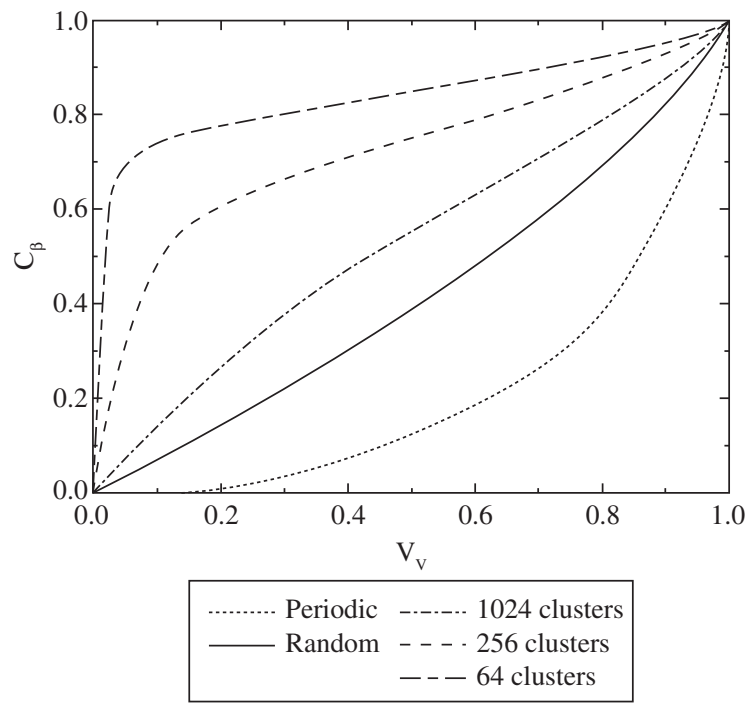

Figure 5. Contiguity, $\mathrm{C}_{\beta}$ against volume fraction, simulated by $\mathrm{CA}$.
2. As shown in Rios et al. ${ }^{14}$, in $2 \mathrm{D}$ simulations the microstructural path developed a pronounced peak at low volume fractions. This did not occur here. Only a slight increase was observed at low volume fractions( See Figure 4.). Of course, the curves in Figure 4 change with clustering but the overall shape remains similar. Therefore, in 3D one might say that in contrast with 2D results the microstructural path is not so sensitive to deviations from randomness;

3. Figure 5 shows that $C_{\beta}$ is a quite good parameter to evaluate deviations from randomness as advocated by Vandermeer ${ }^{12}$. The $\mathrm{C}_{\beta}$ vs. $\mathrm{V}_{\mathrm{v}}$ curve, solid line in Figure 5, for randomly located nuclei divide the $\left(\mathrm{V}_{\mathrm{v}}, \mathrm{C}_{\beta}\right)$ plane in two distinct regions. Below the random curve lies the region in which nuclei deviate from randomness tending to a periodic arrangement. Above the random curve lies the region in which the nuclei tend to cluster. These results are similar to those obtained from 2D simulations; and

4. The interface velocity, calculated from the growth of a single grain and from the global parameters, $\mathrm{S}_{\mathrm{V}}$ and $\mathrm{V}_{\mathrm{V}}$ by means of the Cahn and Hagel equation showed very good agreement. For the single grain, as show in a previous work ${ }^{14}, \mathrm{G}_{\mathrm{CH}}$ (single grain) $\cong 1 / 3$, and for the simulation $\mathrm{G}_{\mathrm{CH}} \cong 0.34$. The only exception was the simulation using nuclei periodically located where there was an slight increase in $\mathrm{G}_{\mathrm{CH}}$ at the later stages of the simulation, $\mathrm{V}_{\mathrm{v}}>0.9$. This shows that the true interface velocity remained constant during all the simulations.

\section{Microstructural Path Analysis}

The microstructural path analysis is carried out in practice by fitting the experimental or simulated equations and comparing the fitted parameters with those calculated for exact cases. Here, this analysis is greatly simplified because it is assumed that the nucleation is site-saturated so that Equations 9 and 18 can be used. Notice that Equations 9 and 18 were derived based on the assumption that nuclei are randomly located in the matrix. This assumption was important because randomness allowed one to use an exact geometrical relationship to take care of the impingement. In practice, if the equations based on the assumption of random nuclei fit the data well one normally concludes that such an assumption is valid and the fitted parameters have a clear meaning with regard to interfacial velocity and number of nuclei per unit of volume. Here, it is shown that this is not entirely correct.

Figure 6 shows the results of three simulations: periodic, random and 64 clusters. Equation 16 is plotted along the random simulation and Equation 9 is force fitted to the periodic and cluster kinetics. Fitting produces correlation coefficients of $\mathrm{R}^{2}=0.999$, indicating very good fit. For periodic nuclei this results in a time dependent apparent velocity that increases with time

$$
\mathrm{G}_{\mathrm{per}}=0.27 \mathrm{t}^{0.13}
$$

For nuclei located in 64 clusters, the apparent velocity decreases with time

$$
\mathrm{G}_{\mathrm{clu}}=0.21 \mathrm{t}^{-0.24}
$$

Equations 21 and 22 show that an analysis solely based on volume fraction could give an erroneous time dependent velocity whereas in all simulations the interface velocity remained constant. For periodic nuclei it could predict that the boundary velocity increases with time. More interesting is the result for the cluster. It predicts that the velocity decreases with time. This is result is quite interesting because it is believed that the velocity decreases with time during recrystallization either owing to concurrent recovery ${ }^{25}$ or the existence of deforma- 


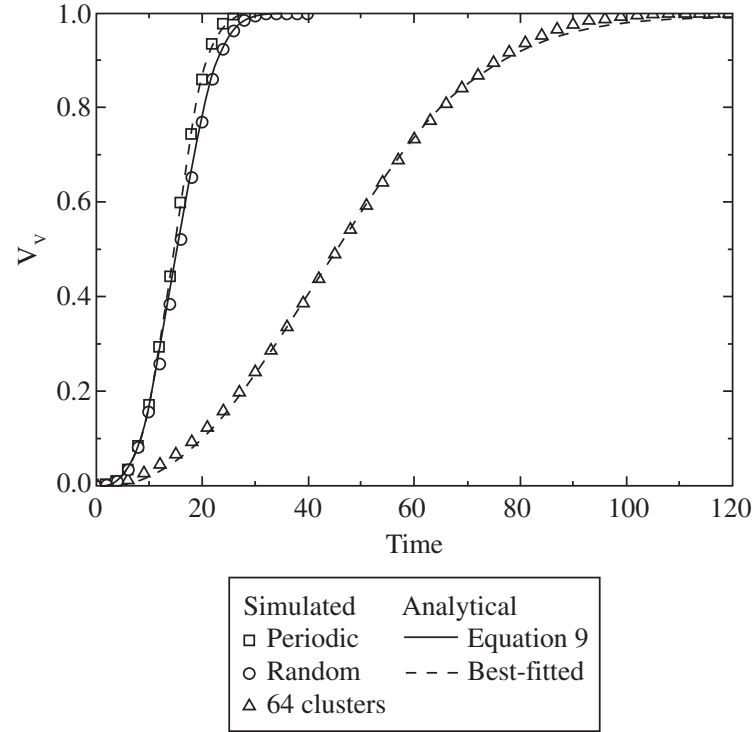

Figure 6. Volume fraction against time, simulated by CA. Simulation data are force fitted by JMAK kinetics, Equation 9 .

tion gradients ${ }^{26}$. The present analysis suggests yet a third possibility: interpreting a non-random microstructure using Equations 9 and 18 , essentially valid for randomly located nuclei, can lead to misleading time dependence on apparent velocity as in Equations 21 and 22. In short, Equation 9 is quite flexible and will give good fit but any data inferred from the fitting parameters is uncertain, unless one can be sure that the nuclei distribution is random. The apparent decrease in growth rate observed here is not real because the interface velocity is kept constant during the simulation. It is purely an artifact arising as a result of the limitations of the mathematical formalism used to analyze the data. This is in full agreement with previous result obtained in 2D simulations.

An indication of randomness is often sought by plotting the microstructural path: $\mathrm{S}_{\mathrm{v}}$ vs. $\mathrm{V}_{\mathrm{v}}$ and using Equation 18 to fit it. Good fit is often taken as an indication that the reaction is site-saturated and that the nuclei distribution is not far from randomness thus validating the above approach. Figure 7 shows this plot for three simulations: periodic, random and 64 clusters. Equation 18 is plotted along the random simulation. Equation 18 is force fitted to the periodic and cluster kinetics curves by allowing $\mathrm{N}_{\mathrm{v}}$ to vary in Equation 18 . From force fitted curves an "apparent" number of nuclei per unit of volume can be calculated.

For periodic nuclei, the apparent number of nuclei per unit of area, $\mathrm{N}_{\mathrm{vper}} \cong 2.0 \times 10^{-4}$, is higher than the value used in the simulation, $\mathrm{N}_{\mathrm{v}} \cong 1.458 \times 10^{-4}$. The correlation coefficient was $\mathrm{R}^{2}=0.99877$, indicating good agreement.

For clustered nuclei, the apparent number of nuclei per unit of volume $\mathrm{N}_{\mathrm{vclus}} \cong 6.591 \times 10^{-6}$, is almost an order of magnitude smaller than the simulation value. The correlation coefficient was still high $\mathrm{R}^{2}=0.97827$. The agreement is still good.

Thus, the microstructural path analysis produced good fit even when there were significant deviations from randomness. In 2D simulations ${ }^{23}$ a similar analysis gave at least some indication that the microstructure was non-random. Here, by contrast, it is clear that just by carrying out such analysis, without any other information, would be very difficult to suspect that nuclei location were non-random. The analysis would then give a wrong idea of the microstructural evolution.

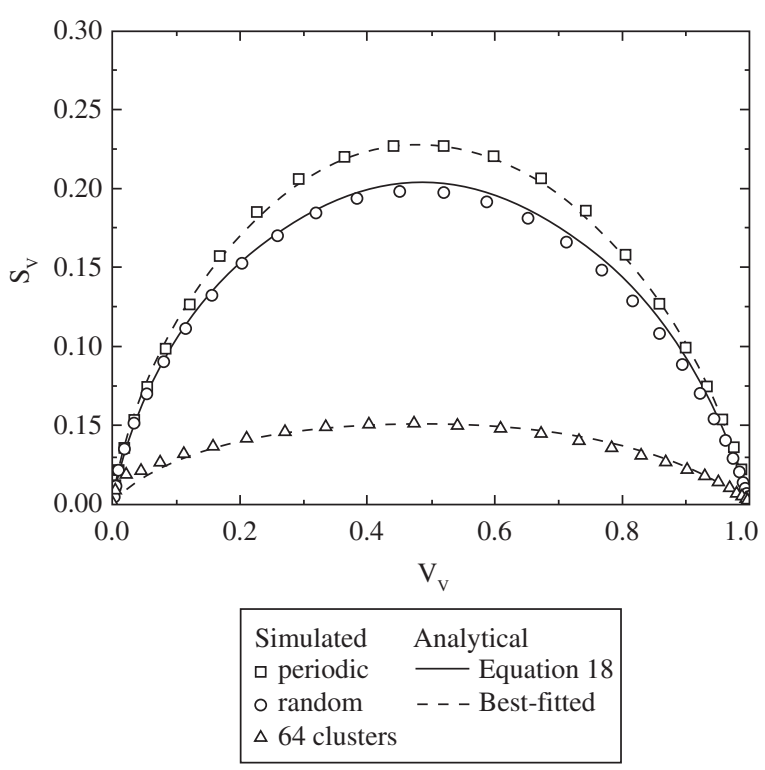

Figure 7. Interfacial area between recrystallized grains and matrix, $S_{\mathrm{v}}$, against volume fraction, simulated by CA. Simulation data are force fitted by Equation 18 , allowing $\mathrm{N}_{\mathrm{V}}$ to vary.

This result shows that for a more reliable analysis it would be highly desirable, if not mandatory, to investigate the possibility of non-random nuclei location. One way would be to use a descriptor able to detect non-randomness such as the contiguity.

The present work shows the relative advantages and disadvantages of computer simulation against the traditional experimental approach. Using computer simulation it was possible to vary the degree of non-randomness. This made possible to infer trends and precisely assess the analytical methodology used in data analysis. This would be extremely difficult to accomplish experimentally.

\section{Summary and Conclusions}

Cellular automata simulation of three dimensional transformations has been carried out to investigate the effect of non-randomness on nuclei location on microstructural evolution. In contrast with 2D simulations, the conventional analysis was much less sensitive to deviations from randomness. Here, conventional kinetic analysis based on the volume fraction against time and the microstructural path based on the $\mathrm{S}_{\mathrm{v}}$ against $\mathrm{V}_{\mathrm{v}}$ plot could not detect non-randomness. This generated misleading time dependent velocity and number of grains per unit of volume. For instance, when nuclei are located in clusters this analysis may erroneously suggest that the velocity decreases with time. Therefore, a more reliable data analysis should include an investigation into the possibility of non-random nuclei location. This is particularly true for 3D transformations. The contiguity, as suggested by Vandermeer ${ }^{12}$, is probably a good choice.

\section{Acknowledgments}

This work was supported by Conselho Nacional de Desenvolvimento Científico e Tecnológico, CNPq, Coordenação de Aperfeiçoamento de Pessoal de Nível Superior, CAPES, and Fundação de Amparo à Pesquisa do Estado do Rio de Janeiro, FAPERJ (Research grant E-26/152397/2002, P. R. Rios).

\section{References}

1. Johnson WA, Mehl RF. Reaction kinetics in processes of nucleation and growth. Transactions AIME. 1939; 135:416-441. 
2. Avrami MJ. Kinetics of phase change I general theory. The Journal of Chemical Physics. 1939; 7(12):1103-1112.

3. Avrami MJ. Kinetics of phase change II transformation-time relations for random distribution of nuclei. The Journal of Chemical Physics. 1940; $8(2): 214-224$

4. Avrami MJ. Kinetics of phase change III granulation, phase change, and microstructure kinetics of phase change. The Journal of Chemical Physics. 1941; 9(2):177-184.

5. Kolmogorov NA. The statistics of crystal growth in metals. Isvestiia Academii Nauk SSSR - Seriia Matematicheskaia. 1937; 1:333-359.

6. DeHoff RT. In: Hansen N, Jensen DJ, Leffers T, Ralph B, editors. Annealing Processes-Recovery, Recrystallization and Grain Growth, Proceedings of the 7th Conference of Ris $\phi$ National Laboratory; 1986, Roskilde, Denmark. Roskilde: Risø National Laboratory; 1986. p. 35-52.

7. Vandermeer RA, Masumura RA, Rath B. Microstructural paths of shapepreserved nucleation and growth transformations. Acta Metallurgica et Materialia. 1991; 39(3):383-389.

8. Cahn JW. The kinetics of grain boundary nucleated reactions. Acta Metallurgica. 1956; 4(9):449-459.

9. Vandermeer RA, Jensen DJ. Effects of nuclei clustering on recrystallization kinetics. Materials Science Fórum. 2004; 467-470:193-196.

10. Sandim HRZ, Martins JP, Padilha AF. Orientation effects during grain subdivision and subsequent annealing in coarse-grained tantalum. Scripta Materialia. 2001; 45(6):733-738.

11. Price CW. Analysis of models for grain-impingement compensation and their effect on recrystallization kinetics. Acta Metallurgica. 1991; 39(8):1807-1816.

12. Vandermeer RA. Microstructural descriptors and the effects of nuclei clustering on recrystallization path kinetics. Acta Materialia. 2005; 53(5):1449-1457.

13. Rios PR, Oliveira JCPT, Oliveira VT, Castro JA. Comparison of analytical models with cellular automata simulation of recrystallization in two dimensions. Materials Research. 2005; 8(3):341-345.

14. Rios PR, Oliveira VT, Pereira LO, Pereira MR, Castro JA. Cellular Automata Simulation of Site-saturated and Constant Nucleation Rate Transformations in Three Dimensions. Materials Research. 2006; 9(2):223-230
15. Hesselbarth HW, Göbel IR. Simulation of recrystallization by cellular automata. Acta Metallurgica et Materialia. 1991; 39(9):2135-2143.

16. Pezzee CF, Dunand DC. The impingement effect of an inert, immobile 2nd phase on the recrystallization of a matrix. Acta Metallurgica et Materialia. 1994; 42(5):1509-1524.

17. Goetz RL, Seetharaman V. Static recrystallization kinetics with homogeneous and heterogeneous nucleation using a cellular automata model. Metallurgical and Materials Transactions A. 1998; 29(9):2307-2321.

18. Marx V, Reher FR, Gottstein G. Simulation of primary recrystallization using a modified three-dimensional cellular automaton. Acta Materialia. 1999; 47(4):1219-1230.

19. Pineda E, Pradell T, Crespo D. Non-random nucleation and the Avrami kinetics. Philosophical Magazine A. 2002, 82(1):107-121.

20. Rios PR, Carvalho JJS, Salazar TC, Paula FVL, Castro JA. Cellular automata simulation of the effect of nuclei distribution on the recrystallization kinetics. Materials Science Forum. 2004, 467-470:659-664.

21. Oliveira JCPT. Simulação do efeito da distribuição dos núcleos na cinética e no caminho microestrutural da recristalização pelo método do autômato celular [unpublished M. Sc. thesis]. Volta Redonda: Universidade Federal Fluminense; 2004.

22. Oliveira VT. Desenvolvimento do Código Computacional para a Simulação da Recrystallização em 3D pelo Método do Autômato Celular. [unpublished M. Sc. thesis]. Volta Redonda: Universidade Federal Fluminense; 2005.

23. Rios PR, Oliveira JCPT, Oliveira VT, Castro JA. Microstructural descriptors and cellular automata simulation of the effects of non-random nuclei location on recrystallization in two dimensions. Materials Research. 2006; 9(2):165-170.

24. Cahn JW, Hagel W. Theory of the Pearlite Reaction. In: Zackay ZD, Aaaronson HI, editors. Decomposition of austenite by Diffusional Processes. New York: Interscience; 1960, p. 131-196.

25. Stüwe HP, Padilha AF, Siciliano F. Competition between recovery and recrystallization. Materials Science and Engineering A. 2002; A333(1-2):361-367.

26. Rios PR. Modeling time dependence of the average interface migration rate in site-saturated recrystallization. Metallurgical and Materials Transactions A. 1997; 28(4):939-946. 